\title{
SOME APPLICATIONS OF WALDHAUSEN'S RESULTS ON IRREDUCIBLE SURFACES
}

\author{
BY \\ C. D. FEUSTEL
}

0. Preliminaries. In this paper we will use a number of results of Waldhausen in [10] to partially answer three questions of Neuwirth posed in [5]. Throughout this paper all spaces will be simplicial complexes and all maps will be piecewise linear. We will denote the boundary, closure, and interior of a set $X$ by bd $(X)$, $\mathrm{cl}(X)$, and int $(X)$ respectively. We denote the closed unit interval by $I$. The author wishes to thank L. S. Husch and N. Max for conversations.

1. Certain maximal subgroups of $\Pi_{1}\left(M^{3}\right)$ and a problem of Neuwirth. In this section we will show that a subgroup of $\Pi_{1}\left(M^{3}\right)$ associated with a closed surface embedded in $M^{3}$ is in some sense maximal. We shall follow Kneser in saying $M^{3}$ is irreducible if every embedded 2-sphere bounds an embedded 3-ball.

THEOREM 1. Let $T_{n}$ and $T_{m}$ be compact closed connected, orientable surfaces. Let $M^{3}$ be an orientable 3-manifold which is irreducible. Let $f$ be an embedding of $T_{n}$ in $M^{3}$ such that $f_{*}: \Pi_{1}\left(T_{n}, y\right) \rightarrow \Pi_{1}\left(M^{3}, x\right)$ is a monomorphism. If $\Pi_{1}\left(T_{m}\right)$ is isomorphic to a subgroup $A \subset \Pi_{1}\left(M^{3}, x\right)$ and $A \supset f_{*}\left(\Pi_{1}\left(T_{n}, y\right)\right)$, then $T_{n}$ is homeomorphic to $T_{m}$ and $f_{*} \Pi_{1}\left(T_{n}, y\right)=A$.

Corollary 1. Let $k$ be a knot. Let $X=S^{3}-k$ and $P$ be a peripheral subgroup of $\Pi_{1}(X)$. If $A$ is an abelian subgroup of $\Pi_{1}(X)$ containing $P$, then $A=P\left({ }^{1}\right)$.

Proof of corollary. This is a consequence of a theorem of Papakyriakopoulos that all abelian subgroups of a knot group are subgroups of $Z \oplus Z$ (Theorem (5.4.2) on p. 56 of [5]) and the fact that $X$ is irreducible.

The proof of the theorem will be divided into four lemmas and a theorem. The desired result is then an easy consequence of 1.4 and 1.5.

We will follow Waldhausen in saying that a surface $S$ contained in a manifold $M$ is incompressible in $M$ if for each component $S_{i}$ of $S$ the natural map from $\Pi_{1}\left(S_{i}\right)$ into $\Pi_{1}(M)$ is an injection and $S_{i}$ is not the 2-sphere.

LEMMA 1.1. Let $M^{3}$ be a compact, connected, orientable, irreducible 3-manifold with incompressible boundary. Let $T_{n}$ be a compact, closed, connected, orientable surface. Let $\Pi_{1}\left(T_{n}\right) \cong \Pi_{1}\left(M^{3}\right)$. Then $M^{3}$ is homeomorphic to $T_{n} \times I$.

Received by the editors May 22, 1969.

( ${ }^{1}$ ) The author has been informed by L. Neuwirth that Corollary 1 has been proved by D. Noga in [11]. 
Proof. Let $g: M^{3} \rightarrow T_{n}$ be a continuous map such that $g_{*}$ is an isomorphism. Then since $\Pi_{2}\left(T_{n} \times I\right)=0$, the lemma follows from 6.1 in [10] by considering $f=g \times 1: M^{3} \rightarrow T_{n} \times I$.

Lemma 1.2. Let $M_{1}$ be a connected 3-submanifold of the 3-manifold M. Assume that $M_{1}$ is a closed subset of $M$ and that $\mathrm{cl}\left(M-M_{1}\right) \cap M_{1}$ is incompressible in $M$. Let l be a loop contained in $M_{1}$. If l is homotopic to a point in $M$, then lis homotopic to a point in $M_{1}$.

Proof. Let $D$ be a disk. We assume that $l$ is homotopic to a point in $M$. Let $f:(D$, bd $(D)) \rightarrow(M, l)$ be a map. Consider $f^{-1}\left(f(D) \cap\right.$ bd $\left.\left(M_{1}\right)\right)=S$ which we may take to be a 1-complex in $D$. Now each loop $k$ in $S$ is nullhomotopic on $D$. Thus $f(k)$ is homotopic to a point in bd $\left(M_{1}\right)$. We can redefine $f$ inside $k$ to lie on bd $\left(M_{1}\right)$. This half of the lemma now follows by redefining $f$ inside of a collection of outer-most loops in $S$.

LEMMA 1.3. Let $T_{n}, T_{m}$ be compact, connected, closed orientable surfaces and $f$ an embedding of $T_{m}$ into $T_{n} \times I$. Then $f_{*}: \Pi_{1}\left(T_{m}\right) \rightarrow \Pi_{1}\left(T_{n} \times I\right)$ is an injection if and only if it is an isomorphism.

Proof. We need only show that if $f_{*}$ is an injection, it is an isomorphism. Assume that $f\left(T_{m}\right)$ does not separate $T_{n} \times\{0\}$ from $T_{n} \times\{1\}$. Then $\left[f\left(T_{m}\right)\right]$ is the boundary of a chain in $C_{3}\left(T_{n} \times I ; Z_{2}\right)$ and $f\left(T_{m}\right)$ bounds a submanifold $X$ of $T_{n} \times I$. Van Kampen's theorem gives the following commutative diagram.

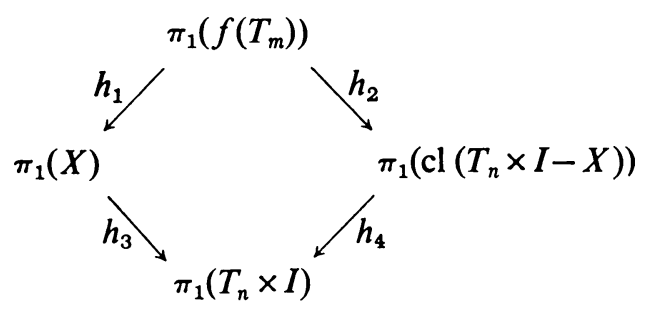

Since $f_{*}$ is an injection, $h_{1}$ and $h_{2}$ are monomorphisms. Since $T_{n} \times I-X$ contains $T_{n} \times\{0\}, h_{4}$ is an epimorphism. It follows from Lemma (4.2) of Brown and Crowell that $h_{1}$ is an isomorphism [2]. Note that $X$ is irreducible since $f\left(T_{m}\right)$ is not contained in a 3-ball. Thus by Lemma $1.1, X$ is homeomorphic to $T_{n} \times I$. It follows that $f\left(T_{m}\right)$ separated $T_{n} \times\{0\}$ from $T_{n} \times\{1\}$.

The desired result follows immediately from Theorem 7.1 in [1].

THEOREM 1.4. Let $M^{3}$ be an irreducible orientable 3-manifold. Let $T_{n}$ be a compact, closed, connected, orientable surface. Let $f: T_{n} \rightarrow M^{3}$ be a map such that

$$
f_{*}: \Pi_{1}\left(T_{n}, y\right) \rightarrow \Pi_{1}\left(M^{3}, x\right)
$$


is a monomorphism. Let $\left(M^{*}, x^{*}, p\right)$ be the covering space of $\left(M^{3}, x\right)$ associated with $f_{*}\left(\Pi_{1}\left(T_{n}, y\right)\right) \subset \Pi_{1}\left(M^{3}, x\right)$. Then there exists an embedding

$$
h:\left(T_{n}, y\right) \rightarrow\left(M^{*}, x^{*}\right)
$$

such that $h_{*}: \Pi_{1}\left(T_{n}, y\right) \rightarrow \Pi_{1}\left(M^{*}, x^{*}\right)$ is an isomorphism.

Proof. Let $g:\left(T_{n}, y\right) \rightarrow\left(M^{*}, x^{*}\right)$ be a p.l. map such that

(1) $g_{*}$ is an isomorphism;

(2) $S=\operatorname{cl}\left\{x \in T_{n}:\{x\} \neq g^{-1} g(x)\right\}$ is a 1-complex.

Let $R$ be a regular neighborhood of $g\left(T_{n}\right)$. We claim that by adding to $R$ and deleting from $R$ 3-balls lying in $M^{*}$, we can obtain a 3-manifold $R_{1}$ satisfying the conditions on $M_{1}$ in Lemma 1.2 , and that there is a map $g^{\prime}$ homotopic to $g$ with image contained in $R_{\mathbf{1}}$.

Let $F$ be a component of bd $(R)$ such that the natural inclusion map from $\Pi_{1}(F) \rightarrow \Pi_{1}\left(M^{*}\right)$ is not an injection.

Suppose $F$ separates $M^{*}$. Let $M_{1}^{*}$ and $M_{2}^{*}$ be the closures of the components of $M^{*}-F$. By Van Kampen's theorem the following commutative diagram exists.

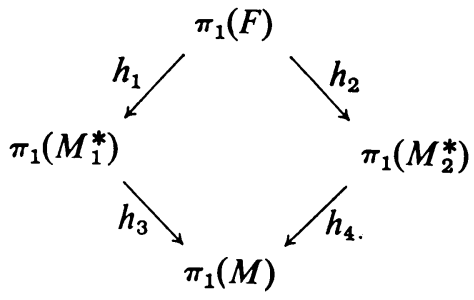

Since $h_{3} h_{1}$ is not one-one it is a consequence of Lemma (4.2) in [2] that either $h_{1}$ or $h_{2}$ is not a monomorphism. By the loop theorem [7], there exists a disk $D$ embedded in $M^{*}$ which meets $F$ only in bd $(D)$ and such that bd $(D)$ is not nullhomotopic on $F$. In case $F$ does not separate $M^{*}$ each cover of it separates the cover of $M^{*}$ associated with the subgroup of $\pi_{1}\left(M^{*}\right)$ each element of which has a representative loop having intersection number zero with $F$.

The existence of the $D$ above follows by an argument similar to the one used above. It follows from a general position argument and then from a cutting argument that we may assume $D$ meets bd $(R)$ in a finite collection of disjoint simple loops no one of which is nullhomotopic on the boundary component of $R$ on which it lies.

It is now clear that we may choose $D$ so that $D \cap$ bd $(R)=b d(D)$ by selecting an innermost loop in $D \cap$ bd $(R)$ bounding a subdisk of $D$. By general position we may assume that $g^{-1}\left(D \cap g\left(T_{n}\right)\right)$ is a 1-complex, possibly empty. We observe that $D$ is contained either in $R$ or in the closure of its complement.

If the former is the case, we remove from $R$ a regular neighborhood of $D$ in $R$. If the latter is the case we add to $R$ a regular neighborhood of $D$ in the closure of the 
complement of $R$. Since the total genus of bd $(R)$ is finite and since the above operation reduces the genus, we can repeat it a finite number of times using a sequence of disks $D_{1} \cdots D_{m}$ until the appropriate maps all become monomorphisms. We denote the manifold obtained thus by $R_{1}$.

We observe that the second disk chosen above may be taken disjoint from the first since the boundary of the second need not meet the first disk and the boundaries of the two disks are unlinked. Thus we may assume that we added and removed 3-balls containing disjoint disks $D_{1}, \ldots, D_{m}$ to and from $R$ to obtain $R_{1}$. We may also assume that $g^{-1}\left(D_{i} \cap g\left(T_{n}\right)\right)$ is a 1 -complex for $i=1, \ldots, m$. We claim that we can find a disjoint collection of disks $E_{1}, \ldots, E_{k}$ contained in $T_{n}$ such that $g^{-1}\left(\bigcup_{i=1}^{m} D_{i} \cap g\left(T_{n}\right)\right)$ is contained in $\bigcup_{i=1}^{k} E_{i}$. This can be seen by the following argument.

Let $c_{i j}$ be a component of $g^{-1}\left(D_{j} \cap g\left(T_{n}\right)\right)$. Since $g_{*}$ is a monomorphism and $g\left(c_{i j}\right) \subset D_{j}$ each loop in the 1-complex $c_{i j}$ is nullhomotopic in $T_{n}$. Let $B_{i j}$ be a regular neighborhood of $c_{i j}$. We may assume that the $B_{i j}$ are mutually disjoint. Now $B_{i j}$ is a compact 2-manifold with boundary. Since each boundary component of $B_{i j}$ is freely homotopic to a loop of $c_{i j}$ each bounds a disk on $T_{n}$. Since $T_{n}$ is not the 2-sphere, one of these disks will contain $B_{i j}$. Let $E_{i j}$ and $E_{u v}$ be disks constructed in the above manner containing $B_{i j}$ and $B_{u v}$ respectively. Now $E_{i j} \cap E_{u v}$ $=\varnothing$ or $E_{i j} \supset E_{u v}$ or $E_{u v} \supset E_{i j}$ and the claim follows.

We may assume that $g\left(\mathrm{bd}\left(E_{i}\right)\right)$ is contained in $R_{1}$. Since $g\left(\mathrm{bd}\left(E_{i}\right)\right)$ is nullhomotopic in $M^{*}$ and $R_{1}$ satisfies the conditions on $M_{1}$ in Lemma 1.2 , bd $\left(E_{i}\right)$ is null homotopic in $R_{1}$. We may redefine $g$ on the $E_{i}$ to obtain a map $g^{\prime}: T_{n} \rightarrow R_{1}$. Since $M$ is irreducible, $\Pi_{2}\left(M^{*}\right)=0$ and $g^{\prime}$ is homotopic to $g$. Clearly we may assume $x^{*}$ is in $R_{1}$.

It follows from Lemma 1.2, that every loop in $R_{1}$ based on $g^{\prime}\left(T_{n}\right)$ can be deformed in $R_{1}$ to lie on $g^{\prime}\left(T_{n}\right)$. Since $g^{\prime}$ is a monomorphism, $\Pi_{1}\left(R_{1}\right) \cong \Pi_{1}\left(T_{n}\right)$. Every 2-sphere in bd $\left(R_{1}\right)$ bounds a homotopy 3 -cell in $M^{*}$ since $M$ is irreducible. We may add these homotopy 3-cells to $R_{1}$ and denote the resulting 3-manifold again by $R_{1}$.

It is a consequence of Lemma 1.1 and "generalization 1 " of J. Milnor's Decomposition Theorem [4], that $R_{1}$ has the homotopy type of $T_{n} \times I$ and that $R_{1}$ has a boundary component homeomorphic to $T_{n}$. The lemma follows.

LEMMA 1.5. Let $M^{3}$ be a connected orientable 3-manifold not necessarily compact. Let $\Pi_{2}\left(M^{3}\right)=0$. Let $f$ and $g$ be embeddings of compact, connected, closed, orientable surfaces $T_{n}, T_{m}$ in $M^{3}$. Let $f_{*}$ be an isomorphism and $g_{*}$ be an injection. Then $g_{*}$ is an isomorphism. Also if $f\left(T_{n}\right) \cap g\left(T_{m}\right)$ is the union of a collection of disjoint simple loops which are not nullhomotopic in $M^{3}$, then there exists a compact neighborhood of $f\left(T_{n}\right) \cup g\left(T_{m}\right)$ with the same homotopy type as $T_{n} \times I$.

Proof. Assume that $f\left(T_{n}\right)$ and $g\left(T_{m}\right)$ are disjoint. We claim that both $f\left(T_{n}\right)$ and $g\left(T_{m}\right)$ separate $M^{3}$. Suppose not. Let $\lambda$ be a loop which meets $f\left(T_{n}\right)$ in a single point. Then $\lambda$ is homotopic to a loop $\lambda_{1}$ contained in a regular neighborhood of $f\left(T_{n}\right)$ 
and not meeting $f\left(T_{n}\right)$. However, the intersection number of $\lambda$ and $f\left(T_{n}\right)$ is one while that of $\lambda_{1}$ and $f\left(T_{n}\right)$ is zero. This is a contradiction and $f\left(T_{n}\right)$ separates $M^{3}$. Similarly $g\left(T_{m}\right)$ separates $M^{3}$. It is easily shown that $f\left(T_{n}\right)$ and $g\left(T_{m}\right)$ are part of the boundary of a connected submanifold $M_{1}$ of $M^{3}$. We may select $M_{1}$ so that bd $\left(M_{1}\right)$ contains no spheres as $\Pi_{2}\left(M^{3}\right)=0$.

It follows from Lemma 1.2 that each loop in $g\left(T_{m}\right)$ is freely homotopic in $M_{1}$ to a loop in $f\left(T_{n}\right)$.

It follows as in the proof of 5.1 in [10] that $M_{1}$ is almost homeomorphic to $T_{n} \times I$, i.e., $M_{1}$ may contain a homotopy 3 -cell which is not a cell. This cell will in fact be bounded by the sphere produced in the proof of (5.1) mentioned above.

If $f\left(T_{n}\right)$ and $g\left(T_{m}\right)$ are not disjoint, we find a homotopy putting them in general position. Thus we may assume that $f^{-1}\left(f\left(T_{n}\right) \cap g\left(T_{m}\right)\right)$ is the union of a family of disjoint simple loops. Similarly $g^{-1}\left(f\left(T_{n}\right) \cap g\left(T_{m}\right)\right)$ is a family of disjoint simple loops. Let $\lambda$ be a simple loop in $f^{-1}\left(f\left(T_{n}\right) \cap g\left(T_{m}\right)\right)$ which is nullhomotopic on $T_{n}$. Then $f(\lambda)$ is nullhomotopic in $M^{3}$; and since $g_{*}$ is a monomorphism, $g^{-1} f(\lambda)$ is nullhomotopic on $T_{m}$. Since $\Pi_{2}\left(M^{3}\right)=0$, we can find a homotopy of $f$ to another embedding with fewer components in $f^{-1}\left(f\left(T_{n}\right) \cap g\left(T_{m}\right)\right)$. It follows that we may take all loops in $f\left(T_{n}\right) \cap g\left(T_{m}\right)$ to be essential in $\Pi_{1}\left(M^{3}\right)$. If $f\left(T_{n}\right) \cap g\left(T_{m}\right)$ is empty we are done by the argument above.

Otherwise let $R$ be a regular neighborhood of $f\left(T_{n}\right) \cup g\left(T_{m}\right)$. Now $R$ is a compact 3-manifold with boundary. Let $F$ be a component of bd $(R)$. If the natural map $i: \Pi_{1}(F) \rightarrow \Pi_{1}\left(M^{3}\right)$ is not a monomorphism we can find a disk $D$ such that bd $(D)=D \cap$ bd $(R)$. Clearly, by a general position and cutting argument, we may assume that $D \cap f\left(T_{n}\right)$ is empty since $f_{*}$ is a monomorphism. By another general position argument we may assume that $D \cap g\left(T_{m}\right)$ is a family of simple loops. Since $g_{*}$ is a monomorphism each loop in $D \cap g\left(T_{m}\right)$ bounds a disk on $g\left(T_{m}\right)$. We denote one such disk by $E_{1}$. Now $E_{1} \cap f\left(T_{n}\right)$ must be empty since $f\left(T_{n}\right) \cap g\left(T_{m}\right)$ contains no simple nullhomotopic loops. It follows that $D \cap g\left(T_{m}\right)$ may be assumed empty. Now either $D$ is contained in $R$ or the closure of the complement of $R$. If the former is the case, we delete from $R$ a regular neighborhood of $D$. This will lower the genus of bd $(R)$. If the resulting manifold has two components we consider the one containing $f\left(T_{n}\right) \cup g\left(T_{m}\right)$. Otherwise we add to $R$ a regular neighborhood of $D$ in the closure of the complement of $R$.

It follows by induction that we may obtain a compact connected 3-manifold $R_{1} \subset M^{3}$ such that for each component $F$ of bd $\left(R_{1}\right)$ the natural map induced by inclusion from $\Pi_{1}(F)$ into $\Pi_{1}\left(M^{3}\right)$ is an injection. It follows from Lemma 1.2. that $\Pi_{1}\left(R_{1}\right) \cong\left(f\left(T_{n}\right)\right)$. If bd $\left(R_{1}\right)$ contains any spheres they bound homotopy 3-cells in $M^{3}$ since $\Pi_{2}\left(M^{3}\right)=0$. We may add these homotopy 3-cells to $R_{1}$ to obtain a compact 3-manifold $R_{2}$ such that $\Pi_{2}\left(R_{2}\right)$ is zero. As in the proof of 1.4, we see that $R_{2}$ is of the homotopy type of $T_{n} \times I$. Thus by Lemma $1.3, g_{*}$ is an isomorphism.

At this point we return to the proof of the theorem.

Let $\left(M^{*}, x^{*}, p\right)$ be the covering of $\left(M^{3}, x\right)$ such that $p_{*} \Pi_{1}\left(M^{*}, x^{*}\right)=A$. Let 
$g:\left(T_{m}, y_{0}\right) \rightarrow\left(M^{3}, x\right)$ be a map such that $g_{*} \Pi_{1}\left(T_{m}, y_{0}\right)=A$. Let $f^{\prime}$ and $g^{\prime}$ be maps covering $f$ and $g$ respectively. Let $g^{\prime \prime}$ be the map whose existence is guaranteed by Lemma 1.4 such that $g_{*}^{\prime \prime}: \Pi_{1}\left(T_{m}, y_{0}\right) \rightarrow \Pi_{1}\left(M^{*}, x^{*}\right)$ is an isomorphism. It follows from 1.5 that $f_{*}^{\prime}$ is an isomorphism since $f_{*}^{\prime}$ is a monomorphism. Since $p_{*} f_{*}^{\prime}=f_{*}$, $f_{*} \Pi_{1}\left(T_{n}, y\right)=A$. Also $f_{*}^{\prime-1} g_{*}^{\prime \prime}: \Pi_{1}\left(T_{m}, y_{0}\right) \rightarrow \Pi_{1}\left(T_{n}, y\right)$ is an isomorphism and thus $T_{n}$ is homeomorphic to $T_{m}$.

2. The relationship of algebraic and geometric splitting of irreducible $M^{3}$. Let $T_{n}$ be a compact, closed, connected, orientable, 2-manifold of genus $n$. Let $M^{3}$ be an irreducible compact, orientable, connected 3-manifold.

We shall say that $M^{3}$ is geometrically split by $T_{n}$ if there exists an embedding $f: T_{n} \rightarrow \operatorname{int}\left(M^{3}\right)$ such that $f_{*}: \Pi_{1}\left(T_{n}\right) \rightarrow \Pi_{1}\left(M^{3}\right)$ is a monomorphism.

We shall say that $M^{3}$ splits algebraically across $T_{n}$ if $\Pi_{1}\left(M^{3}\right)=A *_{\Pi_{1}\left(T_{n}\right)} B$ where $\Pi_{1}\left(T_{n}\right)$ is a proper subgroup of both $A$ and $B$.

THEOREM 2. Let $M^{3}$ be a compact, closed, orientable, 3-manifold and $\Pi_{2}\left(M^{3}\right)=0$. If $M^{3}$ splits algebraically across $T_{n}$ then it is split geometrically by $T_{n}$. Furthermore if $C$ is the group of $\Pi_{1}\left(M^{3}\right)$ associated with $\Pi_{1}\left(T_{n}\right)$ in the algebraic splitting above then we may choose $F$ so that $f_{*}\left(\Pi_{1}\left(T_{n}\right)\right)$ is conjugate to $C$.

Proof. This follows immediately from Satz 1.2 in [9] and Theorem 1.

The theorem above is related to the following problem of Neuwirth found in [5]: "Every knot group contains the group $(a, b ;[a, b])$. This subgroup may be obtained from the natural inclusion of the fundamental group of a nonsingular torus in the knot group. Suppose a knot contains the group of a closed surface of genus $g$. Does there exist a nonsingular closed surface of genus $g$ whose fundamental group is injected monomorphically into the knot group by the natural inclusion?"

3. Coverings of the complement of a link. We shall say that a manifold is in P.C. if every homotopy 3-cell in that manifold is a cell. Let $L=\bigcup_{i=1}^{n} k_{i}$ be a link in $S^{3}$, i.e. the $k_{i}$ are disjoint knots for $i=1, \ldots, n$. Let $X$ be the closure of the complement of a regular neighborhood of $L$.

THEOREM 3. Every covering space of $X$ is in P.C.

Proof. We need only show that the universal cover of $X$ which we denote by $\left(X^{*}, p\right)$ is in P.C. by $(2.3)$ in [3].

Let $S_{i}, i=1, \ldots, l$, be a maximal collection of disjoint 2-spheres embedded in $X$ such that no subcollection of the $S_{i}$ bounds a ball with holes. Let $C_{1}, \ldots, C_{t+1}$ be the closures of the components of $X-\bigcup_{i=1}^{i} S_{i}$. Let $\bar{C}_{i}$ be $C_{i}$ with balls pasted in across all spherical boundary components. It follows from results (2.1), (2.2), and Theorem 2 in [3] that $\bar{C}_{i}$ and $C_{i}$ are in P.C.

We claim that $C_{i}$ for $i=1, \ldots, l+1$ is sufficiently large or a solid torus with finitely many 3-balls removed. The latter is easily seen to be the case whenever $C_{i}$ contains only one trivial knot (it cannot contain two unlinked knots.) The former 
is the case whenever $C_{i}$ contains either a nontrivial link or knot since bd $\left(C_{i}\right)$ $\cap$ bd $(X)$ is incompressible in $C_{i}$.

We claim that each component of $p^{-1}\left(C_{i}\right)$ is in P.C. We observe that each component of $p^{-1}\left(C_{i}\right)$ is simply connected by an easy corollary to Lemma 1.2 since $C_{i} \cap \mathrm{cl}\left(X-C_{i}\right)$ is a family of 2-spheres.

If $C_{\mathfrak{i}}$ is a solid torus with finitely many 3-balls removed, then each component of $p^{-1}\left(C_{i}\right)$ is a universal cover for $C_{i}$ and can be embedded in $E^{3}$.

Otherwise each component of $p^{-1}\left(C_{i}\right)$ can be embedded in a natural way in the universal cover of $\bar{C}_{i}$. Now $\bar{C}_{\mathfrak{i}}$ is aspherical by the sphere theorem [6] and the assumption that the collection of $S_{j}$ is maximal. It follows since both $C_{i}$ and $\bar{C}_{\mathfrak{i}}$ are contained in $S^{3}$ that $\bar{C}_{i}$ is irreducible.

Thus by Theorem 8.1 in [10], the universal cover of $\bar{C}_{i}$ is in P.C. and each component of $p^{-1}\left(C_{i}\right)$ is in P.C. whenever $C_{i}$ is sufficiently large. The claim above is established.

Let $H$ be a homotopy 3-cell in $X^{*}$. Then $H$ meets only finitely many component. of $p^{-1}\left(C_{i}\right)$ for $i=1, \ldots, l+1$. These manifolds meet in 2 -spheres. It is a theorem of Gross [3] that the union of 3-manifolds in P.C. which meet in spheres is in P.C. It follows that $H$ is a cell and $X^{*}$ is in P.C.

\section{On the uniqueness of a certain class of $M_{3}$.}

THEOREM 4. Let $M, M_{1}$, and $M_{2}$ be compact orientable 3-manifolds in P.C. Let $M_{1}$ and $M_{2}$ be irreducible and bd $\left(M_{i}\right)$ both connected and incompressible in $M_{i}$ for $i=1,2$. Let $h$ be a homeomorphism from bd $\left(M_{1}\right)$ to bd $\left(M_{2}\right)$. Let $M$ be closed and $X=M_{1} \cup_{h} M_{2}$. Let $\Pi_{1}(M)=\Pi_{1}(X)$. Then $M$ is homeomorphic to $X$.

Theorem 4 is almost a trivial consequence of 6.5 in [10] and Lemma 4.1. This theorem is interesting mostly because of its relationship to the following problem of Neuwirth [5]: "Suppose the fundamental group of a closed 3-manifold, $M_{1}$ is a free product with amalgamation of two knot groups, $\Pi_{1}\left(S^{3}-k_{1}\right), \Pi_{1}\left(S^{3}-k_{2}\right)$, with the amalgamating subgroup a peripheral subgroup of each knot group. Is this 3-manifold $M=M\left(k_{1}\right) \cup M\left(k_{2}\right)$ where bd $\left(M\left(k_{1}\right)\right)$ and bd $\left(M\left(k_{2}\right)\right)$ are matched by a homeomorphism? $\left(M\left(k_{i}\right)\right.$ denotes the complement in $S^{3}$ of a small tube about $k_{i}$.)" In fact, the author has been informed by L. Neuwirth that the theorem solves the problem as it was originally intended. The difficulty here is that the base point for each of the fundamental groups must have been chosen to lie on the boundary of the closure of the complement of a regular neighborhood of the knot in question.

LEMMA 4.1. Let $M$ be a compact connected orientable 3-manifold. Let $F=\bigcup_{i=1}^{n} F_{i}$ be a system of incompressible disjoint surfaces in $M$. Let $M_{i}, i=1, \ldots, k$, be the closures of the components of $M-F$. Let $M_{i}$ be irreducible for $i=1, \ldots, k$. Then $M$ is irreducible and $\Pi_{2}(M)=0$. 
Proof. Let $S$ be a 2 -sphere embedded in $M$. It follows by a standard general position argument that $F \cap S$ may be taken to be a family of disjoint simple loops. Since the natural map from $\Pi_{1}\left(F_{i}\right)$ into $\Pi_{1}(M)$ is an injection, each loop in $F \cap S$, being nullhomotopic on $S$, must be nullhomotopic in $F$. Let $l$ be a loop in $S \cap F_{1}$ which bounds a disk $D_{1}$ contained in $S$ such that $D_{1} \cap F=l$. Now $l$ bounds a disk $D_{2}$ on $F_{1}$ and $D_{1} \cup D_{2}$ is a sphere which we may assume is embedded in $M_{1}$. By assumption such a sphere bounds a ball in $M_{1}$. Thus it is possible to push $D_{1}$ across this ball isotopically and reduce the number of loops in $S \cap F$. Inductively we see that $S \cap F$ can be assumed empty. It follows that $S$ bounds a ball in one of the $M_{i}$ and also in $M$.

Now $\Pi_{2}(M)=0$, or by the sphere theorem [6] there is a sphere embedded in $M$ which does not bound a ball. This has been shown to be impossible and the lemma follows.

We can now commence the proof of the theorem. We claim that $M$ is irreducible. If not $\Pi_{1}(M)=A * B$ is a nontrivial decomposition of $\Pi_{1}(M)$ which can be obtained from an embedded separating 2-sphere in $M$ that does not bound a ball. We remind the reader that every homotopy 3-cell in $M$ is a ball. Thus $\Pi_{1}(M)=A * B$ and by Knessner's conjecture which was proved by J. Stallings [8] there is a 2-sphere embedded in $X$ which does not bound a ball. This contradicts Lemma 4.1. Thus $M$ is irreducible.

We observe that $M$ and $X$ are trivially boundary irreducible. Since $X$ is sufficiently large by Lemma 1.1.6 in [10] and $\Pi_{1}(X)$ is isomorphic to $\Pi_{1}(M)$, it follows from (6.5) in [10] that there is a homeomorphism $f$ from $X$ to $M$ which induces the above isomorphism. We point out that Waldhausen's condition that the isomorphism from $\Pi_{1}(M)$ to $\Pi_{1}(X)$ preserve the peripheral structure is trivially satisfied since $M$ and $X$ are closed.

\section{REFERENCES}

1. E. M. Brown, Unknotting in $M^{2} \times I$, Trans. Amer. Math. Soc. 123 (1966), 480-505. MR 33 \#6640.

2. E. M. Brown and R. H. Crowell, The augmentation subgroup of a link, J. Math. Mech. 15 (1966), 1065-1074. MR 33 \#4920.

3. J. Gross, Manifolds in which the Poincaré conjecture is true, Trans. Amer. Math. Soc. 142 (1969), 177-189.

4. J. Milnor, A unique decomposition theorem for 3-manifolds, Amer. J. Math. 84 (1962), 1-7. MR 25 \#5518.

5. L. P. Neuwirth, Knot groups, Ann. of Math. Studies no. 56, Princeton Univ. Press, Princeton, N. J., 1965. MR 31 \#734.

6. C. D. Papakyriakopoulos, On Dehn's lemma and the asphericity of knots, Ann. of Math. (2) 66 (1957), 1-26. MR 19, 761.

7. J. Stallings, On the loop theorem, Ann. of Math. (2) 72 (1960), 12-19. MR 22 \#12526.

8. - Kneser's conjecture, mimeographed notes by J. Hemple, Rice University. 
9. F. Waldhausen, Gruppen mit Zentrum und 3-dimensionale Mannigfaltigkeiten, Topology 6 (1967), 505-517. MR 38 \#5223.

10. - On irreducible 3-manifolds which are sufficiently large, Ann. of Math. (2) 87 (1968), 56-88. MR 36 \#7146.

11. Dieter Noga, Uber den Aussenraum von Produktknoten und die Bedeutung der Fixgruppen, Math. Z. 101 (1967), 131-141. MR 36 \#2137.

\section{UNIVERSITY OF GEORGIA,}

Athens, Georgia 30601 